\title{
CARACTERIZACIÓN DE MACROINVERTEBRADOS BENTÓNICOS DE DOS RAMALES ESTUARINOS AFECTADOS POR LA ACTIVIDAD INDUSTRIAL, ESTERO SALADO-ECUADOR
}

\author{
BENTHONIC MACROINVERTEBRATES CHARACTERIZATION ON TWO ESTUARINE \\ BRANCHES AFFECTED BY INDUSTRIAL POLLUTION, ESTERO SALADO-ECUADOR
}

Maritza Cárdenas-Calle y James Mair

\begin{abstract}
RESUMEN
El propósito de este estudio fue evaluar la composición, abundancia y diversidad de los macroinvertebrados en dos zonas afectadas por aguas residuales industriales y domésticas en dos ramales estuarinos en la ciudad de Guayaquil: Aventura Plaza y la Universidad de Guayaquil. Se realizaron tres muestreos por sitio, utilizando una draga Van Veen de 0,1 m durante la marea baja en septiembre de 2012. No se encontraron diferencias significativas entre las varianzas de la riqueza y abundancia de especies, registrándose la mayor abundancia en el ramal estuarino cercano a la Universidad de Guayaquil, donde el taxón más abundante fueron los oligoquetos y donde se presentó la más alta temperatura y mayor salinidad. Los sitios estudiados fueron muy pobres y poco diversos debido posiblemente a las emisiones de aguas residuales de uso doméstico e industrial que alteran parámetros claves para la supervivencia de especies estuarinas como la salinidad, la temperatura y el pH.
\end{abstract}

PALABRAS CLAVES: contaminación difusa, Estero Salado, macroinvertebrados

\begin{abstract}
The purpose of this study was to assess composition, abundance and diversity of macroinvertebrates on two areas affected by industrial and domestic wastewater into two estuarine branches in the city of Guayaquil: Aventura Plaza and the Universidad de Guayaquil. Three samplings per site were performed using a Van Veen grab from $0.1 \mathrm{~m}$ at low tide in September 2012. No statistical differences were detected between in the variances of richness and abundance species, the most abundant was the nearest branch to estuarine Universidad de Guayaquil, where the most abundant taxa were Oligochaeta, presented the higher temperature and higher salinity. While the study sites were very poor and few diverse, could be related to emissions from municipal sewage and industrial uses that changed key parameters for the survival of estuarine species such as salinity, temperature and $\mathrm{pH}$.
\end{abstract}

KEYWORDS: diffuse pollution, diversity, Estero Salado, macroinvertebrates

\section{INTRODUCCIÓN}

Los manglares constituyen uno de los ecosistemas acuáticos de mayor productividad biológica, esto es unas 20.000 Kcal.m ${ }^{-2}$.año ${ }^{-1}$ (Montaño y Sanfeliú, 2008). Se encuentran en las zonas tropicales y subtropicales y albergan una gran diversidad de especies de fauna y flora (Márquez y Jiménez, 2002), que se han adaptado a vivir en ambientes salinos (Bouillon, 2011), tales como mangles, moluscos, crustáceos, equinodermos, tunicados y otros invertebrados que están bien adaptados a cambios de mareas, salinidad, temperatura, suelos anaeróbicos y que sustentan la alimentación, crecimiento, reproducción y supervivencia de especies acuáticas y niveles tróficos superiores, incluyendo al hombre.

En el Ecuador los manglares forman parte de una variedad de hábitats que se extienden a lo largo de aproximadamente 2900 km de línea de costa (Boothroyd 
et al, 1994), siendo uno de los estuarios de mayor importancia el Estero Salado por formar parte del Golfo de Guayaquil, situado en la provincia del Guayas y representado por seis especies de mangle, de los cuales tres son conocidos comúnmente como mangles rojos Rhizophora mangle, $R$. harrisonii, $R$. racemosa, Conocarpus erectus (mangle jelí), Laguncularia racemosa (mangle blanco) (Valverde y Pérez, 2012) y Avicennia germinans (mangle negro) (Pérez, 2012), así como otras formaciones vegetales hidrohalófitas (ej. Salicornia fruticosa, Cryptocarpus pyriformes, Batis maritima). Las aguas del Golfo son biológicamente fértiles y soportan importantes pesquerías artesanales del Golfo de Guayaquil como las de peces como el barrilete (Katsuwonus pelamis), aleta amarilla (Thunnus albacares) (Stevenson, 1981); moluscos como Ostrea columbiensis (Mora y Reinoso, 1981), Anadara tuberculosa, A. similis, Mytella guyanensis, M. strigata; y crustáceos como el cangrejo Ucides occidentalis.

El Golfo de Guayaquil, es considerado el estuario más grande de la costa sudamericana del Pacífico. Posee una extensión de $13701 \mathrm{~km}^{2}$ (11711 km² de superficie de agua y $1990 \mathrm{~km}^{2}$ de islas e islotes) (CAAM, 1996; Stevenson, 1981). El Golfo está dividido naturalmente en estuario exterior e interior. El Estero Salado y el sistema del río Guayas forman parte del estuario interior, el Estero Salado se inicia en la ciudad de Guayaquil y se extiende hacia el Sur-Oeste, hasta el Canal del Morro (Cruz, 1992) y tiene una extensión de 74 km (Stevenson, 1981).

Entre los principales cambios que ha sufrido el Estero Salado debido a su cercanía a Guayaquil se encuentran alteraciones en sus características físicas, químicas y biológicas tales como: la reducción de sus ramales, falta de renovación de sus aguas (Holden, 1978), incremento de aguas residuales de uso doméstico sin tratamiento previo (EMAG, 1978), aumento de concentraciones de nitrógeno, fósforo, bacterias fecales y virus (Twilley, 1989). Y una amplia gama de químicos persistentes que se descargan al estero (Pires, 2000) como: hidrocarburos, aceites, grasas (Cárdenas, 2010; Rodríguez, 2005; Thiakos, 2000), blanqueadores, metales pesados como Al, As, Cr, Ni, Zn y Pb (Fernández-Cadena et al., 2014), detergentes, cloraminas (CAAM, 1996), afectando negativamente la calidad del agua del Estero así como la composición, abundancia, diversidad y distribución de la biota acuática planctónica y bentónica (Cárdenas, 2010; Hidroestudios, 2003).
Entre los principales estudios realizados sobre macroinvertebrados en el Estero Salado, se encuentran descripciones de moluscos realizadas por Olsson (1961) y Keen (1971), listados de especies de la fauna sublitoral bentónica del Estero Salado Interior incluyendo moluscos, anélidos, crustáceos, foraminíferos (Cruz, et al., 1980); estado de las poblaciones de ostiones en tres zonas del estuario interior del Golfo de Guayaquil (Mora y Reinoso, 1981); bivalvos del Golfo de Guayaquil (Cruz, 1983); estudios descriptivos de especies de bivalvos en el Estero Salado y el Estero Cascajal (Cruz, 1986); estado del recursos malacológico de la zona infralitoral del Golfo de Guayaquil (Cruz, 1992); malacofauna existente alrededor de la ciudad de Guayaquil durante 2003 (Cruz, 2003).

Otros estudios han incluido el análisis del efecto de la contaminación hidrocarburífera en la estructura comunitaria de macroinvertebrados bentónicos en el Estero Salado (Cárdenas, 2010); así como el estudio de los niveles de coliformes totales y Escherichia coli en bivalvos de interés comercial (Siguencia, 2010); el levantamiento de la batimetría y caracterización del sedimento del Estero Salado y su relación con la diversidad macrobentónica (INCOAR, 2012), entre otros estudios.

Entre los factores que inciden en la distribución de las comunidades macrobentónicas se encuentran la salinidad, tipo de sustrato, corrientes, disponibilidad de nutrientes (Cruz et al., 1980). En este estudio se describe los parámetros de temperatura, salinidad, pH del agua y sedimentos; así como la estructura (composición, abundancia, diversidad y uniformidad) de macroinvertebrados en dos zonas afectadas por aguas residuales industriales y domésticas cercanas a la ciudad de Guayaquil: Aventura Plaza y la Universidad de Guayaquil.

\section{MATERIALES Y MÉTODOS}

\section{Descripción del área de estudio}

Los sitios de estudio fueron dos ramales estuarinos que ingresan a la ciudad de Guayaquil denominados por las autoridades de control ambiental como tramo B y $\mathrm{C}$, estos forman parte de la zona I que incluyen áreas de asentamientos humanos provistos de servicios de 
agua potable, alcantarillado y servicios urbanísticos (Figura 1), pero con alta afectación de aguas residuales industriales y de uso doméstico (Pires, 2000). Las estaciones de muestreo comprendieron los ramales colindantes al Centro Comercial Aventura Plaza (Tramo B) con las coordenadas latitud: $2^{\circ} 10^{\prime} 16.37^{\prime \prime} \mathrm{S}$ y longitud: $79^{\circ} 54^{\prime} 47.57^{\prime \prime} 0$ y la Universidad de Guayaquil (Tramo C) con las coordenadas geográficas latitud: $2^{\circ} 10^{\prime} 54.15^{\prime \prime} \mathrm{S}$ y longitud: $79^{\circ} 54^{\prime} 03.39^{\prime \prime} \mathrm{O}$. El área de estudio pertenece a una región muy seca tropical
Valverde y Pérez, 2012), tiene una precipitación media anual $1107,7 \mathrm{~mm}$, con una temperatura de $25,3^{\circ} \mathrm{C}$, una humedad relativa del $75 \%$, y predominan los sedimentos limosos (Cruz, 1986), variando su textura entre limoarcilloso y/o arcillo-limoso (Ayarza et al., 1993) y se encuentran dos especies de mangles (Rhizophora harrisonii, Laguncularia racemosa) en Aventura Plaza y tres especies (Rhizophora mangle, Avicennia germinans y Conocarpus erectus) en la zona de la Universidad de Guayaquil (Pérez, 2012).

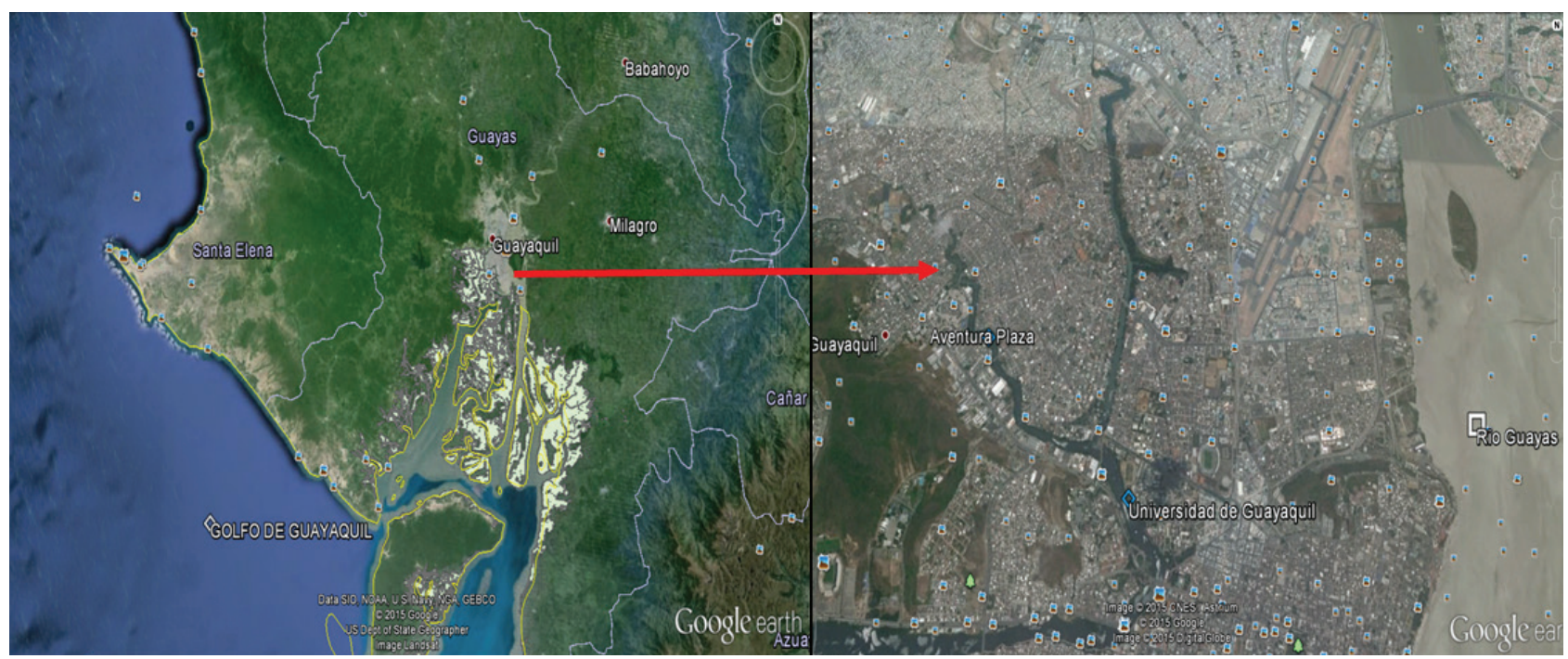

Figura 1. Ubicación de los sitios de muestreo: Aventura Plaza y Universidad de Guayaquil, ramales estuarinos del Estero Salado que ingresan a la ciudad de Guayaquil (Fuente: Modificado de Google Earthº 2014)

\section{Fase de campo}

La valoración de la composición, abundancia, diversidad y uniformidad de las comunidades de macroinvertebrados bentónicos se realizó en septiembre de 2012 durante la época seca. Las muestras fueron extraídas del sedimento mediante el uso de la draga Van Veen, de $0.1 \mathrm{~m}^{2}$ de área de mordida durante la marea baja en la zona intermareal, en cada estación de muestreo se obtuvieron tres réplicas que fueron tamizada en un tamiz de ojo de malla de $1 \mathrm{~mm}$, especialmente usado para retener macroinvertebrados (FAO, 1981), 120 los organismos retenidos en el tamiz fueron fijados y preservados con formol al $10 \%$ para su posterior identificación en el laboratorio. Adicionalmente se registraron las variables de salinidad, temperatura, $\mathrm{pH}$ de agua y sedimento.

\section{Fase de laboratorio}

Los organismos fueron separados del sedimento mediante el uso de un esteromicroscopio marca AmScope modelo SM-17z2-PL-9M, las muestras fueron transferidas a frascos de $25 \mathrm{cc}$ con formol al $10 \%$ y fueron identificadas hasta el nivel taxonómico más bajo posible usando claves taxonómicas específicas tales como (Cruz y Jiménez, 1994; Domínguez y Fernández, 2009; Holthius, 1951; Roldan, 1988). Las muestras 
fueron analizadas en el laboratorio de biología de la Escuela de Ciencias Ambientales de la Universidad de Especialidades Espíritu Santo.

\section{Análisis estadístico}

La estructura comunidad macrobentónica se determinó mediante el uso del índice de diversidad de ShannonWeaver $\left(\mathrm{H}^{\prime}\right)$, siguiendo la fórmula: $\mathrm{H}^{\prime}=-\sum$ pi. $\log _{2}$ pi. Donde pi es la abundancia proporcional de la especie $\mathrm{i}$, donde pi $=($ ni / $\mathrm{N})$, número de individuos de una especie dividido para el número total de individuos de todas las especies; por lo que el índice puede ser interpretado como la relación entre el número de especies $S$ y su abundancia relativa (Shannon y Weaver, 1963). Además se calculó la riqueza de especies (d) que es número de especies representadas en la muestra y la uniformidad o equitabilidad $\left(\mathrm{J}^{\prime}\right)$ que fue determinada mediante el índice de Pielou $\left(\mathrm{J}^{\prime}\right)$ mediante la fórmula $\mathrm{J}^{\prime}=\mathrm{H}^{\prime} / \mathrm{H}$ Máxima. El índice de diversidad, la riqueza de especies, y la equitabilidad fueron calculados mediante el uso del menú DIVERSE del software PRIMER 6.0 (Plymouth Routines in Multivariate Ecological Research).

Se efectuaron pruebas no paramétricas debido a que los datos no mostraron una distribución uniforme. Para ello se generó una matriz de similitud utilizando el índice de Bray-Curtis sobre datos transformados con la opción raíz cuarta para quitar peso a las especies dominantes (Clark y Warwick, 1994). Se realizó un Análisis de Escalamiento Multidimensional (Multiple Dimensional Scaling, MDS) para mostrar posibles relaciones entre las abundancias de los macroinvertebrados entre las estaciones.

Para poner a prueba la hipótesis de que existen diferencias entre los ensambles de macroinvertebrados asociados a las diferentes estaciones, se realizó un análisis de similitud (ANOSIM, nivel de significancia del $1 \%$ ) de dos vías a la diversidad, equidad y riqueza para determinar si existían diferencias significativas entre los sitios de muestreo. El ANOSIM es un análisis de permutaciones y provee una herramienta para poner a prueba si existen diferencias significativas entre distintos grupos de unidades de muestreo, es análogo al análisis de varianza de 1 y 2 vías (Anova de 1 y 2 vías) (Clarke y Gorley, 2006) y se usó para determinar diferencias significativas entre las estaciones. Para este caso se trabajó con un valor de significancia de $\mathrm{p}<0,001$. El valor del estadístico de esta prueba ( $\mathrm{R}$ global) es una medida comparativa del grado de separación entre los grupos: $\mathrm{R}=1$ implica que todas las muestras dentro de un grupo son más parecidas entre sí que a cualquier otra muestra de otro grupo, mientras que $\mathrm{R}=0$, implica poca o nula segregación dentro del grupo y $\mathrm{R}<0,5$ indica solapamiento entre los grupos (Clark y Warwick, 2001). Se usó el software PRIMER 6.0 para realizar los análisis de diversidad, NMDS y ANOSIM. Además se realizó un análisis de componentes principales para determinar la relación entre las variables ambientales y las variables biológicas mediante el uso del programa Infostat.

Para establecer si había diferencias significativas de las variables ambientales entre las localidades se realizó una comparación por parejas Mann Whitney (Zar, 1996) para la salinidad, temperatura, $\mathrm{pH}$ de agua y sedimento mediante el programa Statgraphics Plus y para analizar las interrelaciones entre las variables ambientales y las variables biológicas se aplicó el análisis de componentes principales mediante el uso del programa estadístico Infostat.

\section{RESULTADOS}

\section{Variables ambientales}

La salinidad promedio fue de 14,5 UPS; la localidad Aventura Plaza presentó la menor salinidad cuyo valor fue de 14 UPS, en comparación con la estación Universidad de Guayaquil que presentó 15 UPS. La temperatura fue similar en ambas estaciones con una temperatura promedio de $26,1{ }^{\circ} \mathrm{C}$ decreciendo la temperatura ligeramente a $25,9^{\circ} \mathrm{C}$ en la Universidad de Guayaquil, esto podría estar relacionado a la variación normal de la temperatura a lo largo del día. El pH promedio del agua fue neutro $(7,4)$ siendo ligeramente alcalino en la Universidad de Guayaquil con un valor de 7,6 mientras que en la estación de Aventura Plaza mantuvo la neutralidad con un valor de 7,1.

El pH del sedimento en ambas estaciones se mantuvo neutro con un $\mathrm{pH}$ promedio de 7,1 este valor fue inferior al pH promedio registrado en el agua (Tabla 1). No existieron diferencias significativas entre las variables ambientales analizadas para las estaciones de estudio (Mann-Whitney U-test, $\mathrm{p}<0,05$ ). 
Tabla 1. Variables ambientales y análisis de comparación de medianas usando la prueba de Mann-Whitney U-test.

\begin{tabular}{ccccc}
\hline \multirow{2}{*}{ Variable } & \multicolumn{2}{c}{ Estación } & Valor $\mathbf{p}$ & U- test \\
\cline { 2 - 3 } & Aventura Plaza & Univ. De Guayaquil & & \\
\hline Salinidad & 14 & 15 & $<0,05$ & 0,1 \\
Temperatura & 26,1 & 25,9 & $<0,05$ & 0,66 \\
pH agua & 7,1 & 7,6 & $<0,05$ & 0,66 \\
pH sedimento & 7 & 7,1 & $<0,05$ & 0,86 \\
\hline
\end{tabular}

\section{Variables biológicas}

La comunidad macrobentónica estuvo representada por 461 individuos, representados por 6 especies, 6 géneros, 8 familias y 5 clases del zoobentos, distribuidas en el sedimento y representadas por 3 phyla: Arthropoda, Annelida y Mollusca. Los anélidos fueron el grupo más abundante, con el $76 \%$, seguido de los artrópodos con el $16 \%$ y los moluscos con el $8 \%$ (Figura 2).

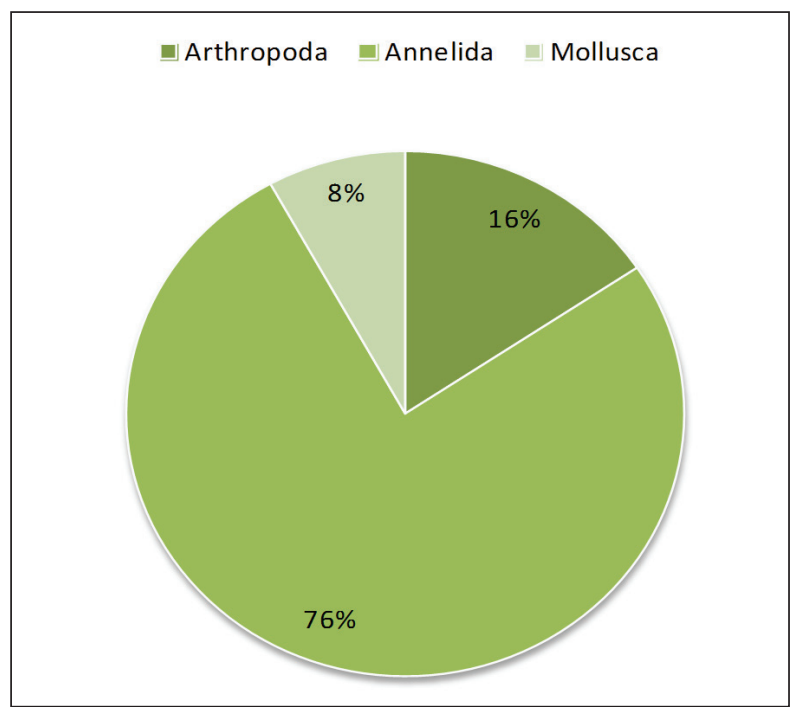

Figura 2. Composición porcentual de la fauna macrobentónica de dos ramales del Estero Salado influenciados por actividades humanas en Guayaquil, Ecuador.

122

La estación con mayor abundancia de organismos fue la Universidad de Guayaquil con 379 individuos el grupo taxonómico más abundante fue el de los oligoquetos representados por familia Enchitraeidae (Tabla 2). Mientras que en la estación Aventura Plaza se registró la menor abundancia de macroinvertebrados con 82 individuos representados por la clase Insecta, especialmente por los dípteros de la familia Psychodidae con el género Pericoma con 62 individuos, con una menor abundancia se registró a las familias Dolichopodidae (7 individuos) y Poduridae (2 individuos) con la especie Podura aquatica y una pupa no identificada.

Los moluscos estuvieron representados por las clases gastropoda y bivalvia, siendo los gasterópodos los más abundantes, pertenecientes a la familia Hydrobiidae representado por el género Heleobia, la familia Thiaridae con la especie Melanoides tuberculata y la familia Ellobiidae con la especie Melampus cf. carolianus. Los gasterópodos se encontraron en mayor abundancia en la estación Universidad de Guayaquil (28 individuos) en comparación con la estación Aventura Plaza que sólo presentó 7 individuos del género Heleobia. Los bivalvos estuvieron representados por la familia Corbiculidae por el género Polymesoda inflata y un espécimen no identificado.

El análisis de la abundancia y riqueza de especies permitió distinguir un patrón espacial que establece que no hay similitud entre las comunidades macrobentónicas presentes en las localidades de estudio. El valor de estrés obtenido fue de 0 (Figura 3), ratificando que se trata de dos comunidades distintas, en la cual la estación Aventura Plaza se caracterizó por la presencia de insectos dípteros del género Pericoma mientras que los oligoquetos estuvieron presentes en la estación Universidad de Guayaquil. 
Tabla 2. Macroinvertebrados registrados en los sedimentos de los ramales estuarinos de Aventura Plaza y la Universidad de Guayaquil en el Estero Salado durante septiembre de 2012

\begin{tabular}{|c|c|c|c|c|c|c|c|}
\hline \multirow{3}{*}{ RÉPLICAS } & & \multicolumn{6}{|c|}{ SITIOS } \\
\hline & & \multicolumn{3}{|c|}{ Aventura Plaza } & \multicolumn{3}{|c|}{ Universidad de Guayaquil } \\
\hline & & A & B & $\mathrm{C}$ & A & B & $\mathrm{C}$ \\
\hline \multicolumn{8}{|l|}{ Phylum Arthropoda } \\
\hline \multirow[t]{3}{*}{ Clase Insecta } & Pericoma sp. Walker & 32 & 6 & 24 & 0 & 0 & 0 \\
\hline & Podura aquatica Linnaeus & 0 & 0 & 2 & 0 & 0 & 0 \\
\hline & Dolichopodidae & 2 & 4 & 1 & 0 & 0 & 0 \\
\hline \multicolumn{8}{|l|}{ Phylum Annelida } \\
\hline \multirow[t]{2}{*}{ Clase Oligochaeta } & Enchitraeidae & 0 & 0 & 0 & 147 & 67 & 131 \\
\hline & Oligochaeta & 1 & 2 & 0 & 0 & 0 & 0 \\
\hline Clase Polychaeta & No determinado & 0 & 0 & 0 & 4 & 0 & 0 \\
\hline \multicolumn{8}{|l|}{ Phylum Mollusca } \\
\hline \multirow[t]{3}{*}{ Clase Gastropoda } & Melampus cf, carolianus Lesson & 0 & 0 & 0 & 0 & 2 & 0 \\
\hline & Melanoides tuberculata O.F. Müller & 0 & 0 & 0 & 0 & 2 & 0 \\
\hline & Heleobia sp. Stimpson & 0 & 7 & 0 & 5 & 0 & 19 \\
\hline \multirow[t]{2}{*}{ Clase Bivalvia } & Polymesoda inflata Philippi & 0 & 0 & 0 & 0 & 1 & 0 \\
\hline & Bivalvo no determinado & 0 & 0 & 0 & 1 & 0 & 0 \\
\hline
\end{tabular}

El análisis de diversidad de Shannon -Weaver demostró que los dos sitios de estudio presentaron una diversidad biológica muy baja, con un índice promedio de 0,23 bits. org $^{-1}$, siendo la estación de Aventura Plaza la de mayor diversidad promedio con 0,318 bits. $\operatorname{org}^{-1}$, mientras que la Universidad Guayaquil presentó una menor diversidad promedio de especies de 0,1451 bits.org-1 (Tabla 3). La riqueza promedio de las estaciones muestreadas fue 0,66 siendo la riqueza promedio de la estación Aventura Plaza la mayor con 0,8348 en comparación con la estación de la Universidad de Guayaquil que presentó un valor de 0,4981 , para ambos casos fue muy baja y la equitabilidad promedio fue 0,43 siendo la estación Aventura Plaza en donde se presentó el valor más alto de equitabilidad $(0,52)$ en comparación con la estación Universidad de Guayaquil.

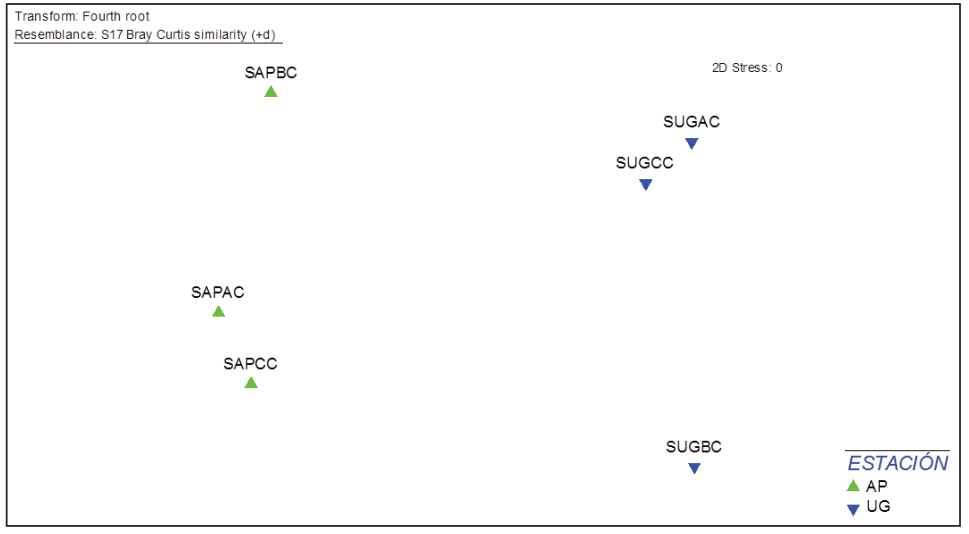

Figura 3. Análisis de ordenamiento (n-MDS) de las estaciones Aventura Plaza y Universidad de Guayaquil. SAPAC (septiembre- estación Aventura Plaza- réplica A- caracterización); SAPBC (septiembre- estación Aventura Plaza- réplica B- caracterización); SAPCC (septiembre- estación Aventura Plaza- réplica C- caracterización); SUGAC (Septiembre- estación Universidad de Guayaquil- réplica A- caracterización); SUGBC (septiembre- estación Universidad de Guayaquil- réplica B - caracterización); SUGCC (septiembre- estación Universidad de Guayaquil- réplica C - caracterización) 
Tabla 3. Indices de diversidad de Aventura Plaza y la Universidad de Guayaquil durante la caracterización de los ramales estuarinos del Estero Salado realizados en septiembre de 2012

\begin{tabular}{cccccc}
\hline Estaciones & Réplicas & $\begin{array}{c}\text { No especies } \\
(\boldsymbol{S})\end{array}$ & $\begin{array}{c}\text { Riqueza de } \\
\text { especies }(\boldsymbol{d})\end{array}$ & $\begin{array}{c}\text { Indice } \\
\text { Shannon - Weaver } \\
\left(\mathbf{H}^{\prime}\right)\end{array}$ & $\begin{array}{c}\text { Equitabilidad } \\
\left(\boldsymbol{J}^{\prime}\right)\end{array}$ \\
\hline \multirow{3}{*}{ Aventura Plaza } & SAPA & 3 & 0,5625 & 0,1507 & 0,3159 \\
& SAPB & 5 & 1,3352 & 0,6213 & 0,8889 \\
Universidad de & SAPC & 3 & 0,6068 & 0,1822 & 0,3819 \\
Guayaquil & SUGA & 4 & 0,5933 & 0,1290 & 0,2143 \\
& SUGB & 4 & 0,7015 & 0,1413 & 0,2348 \\
\hline
\end{tabular}

Réplicas: $\mathrm{S}=$ Septiembre, $\mathrm{AP}=$ Aventura Plaza, $\mathrm{UG}=$ Universidad de Guayaquil, A-B-C $=$ réplicas.

Las fluctuaciones espaciales de los indicadores estructurales de la comunidad se muestran en la figura 4. La riqueza de especies, el índice de diversidad y la equitabilidad variaron en las localidades del estudio. El análisis de ANOSIM demostró que no existieron diferencias significativas $(R=0,17 ; p<0,001)$ entre las abundancias de los invertebrados y entre las estaciones de muestreo.

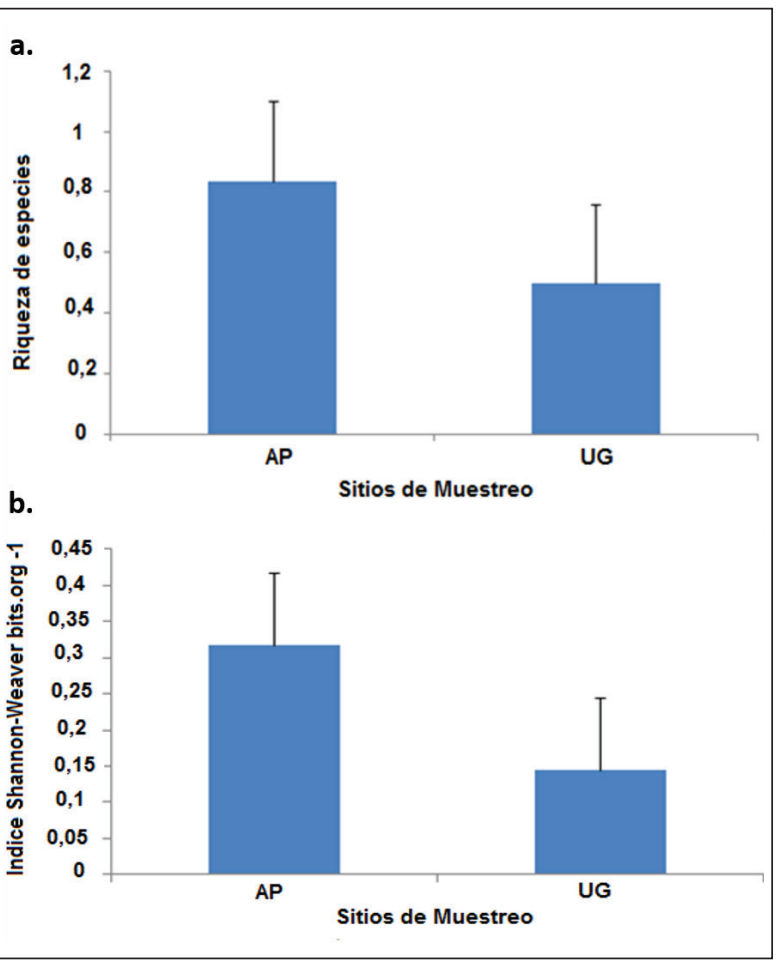

Figura 4. Promedios (barras) y desviaciones estándar (líneas) de la riqueza (izquierda) y diversidad (derecha) de los macroinvertebrados bentónicos registrados en los dos ramales del Estero Salado.
En relación a la variabilidad de la riqueza, abundancia, diversidad y distribución de invertebrados en los sitios de estudio y su relación con las variables ambientales registradas se observó que existe una asociación positiva entre la abundancia de organismos con el pH del agua y la salinidad en la estación Universidad de Guayaquil. Mientras que en la estación Aventura Plaza esta misma asociación se registró entre las variables temperatura y el índice de diversidad (Figura 5).

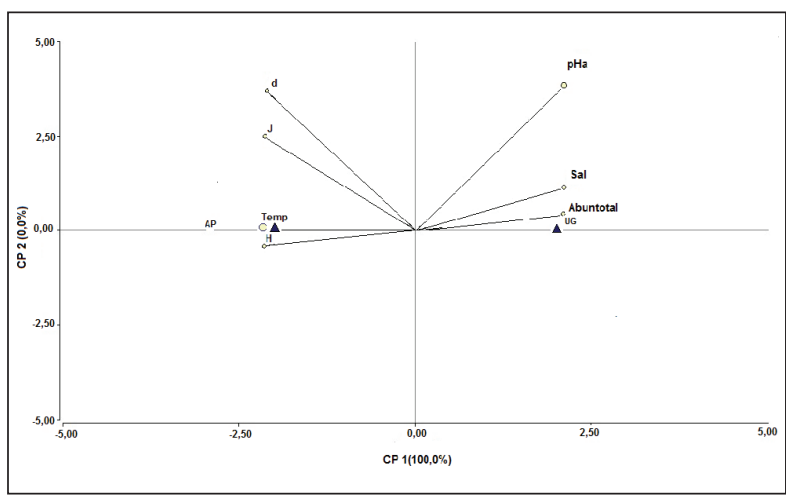

Figura 5. Análisis de Componentes principales entre las variables ambientales y biológicas de los ramales estuarinos Aventura Plaza (AP) y Universidad de Guayaquil (UG) del Estero Salado registrados durante septiembre de 2012

\section{DISCUSIÓN}

La riqueza de la fauna de macroinvertebrados bentónicos del Estero Salado puede ser evaluada comparándose con el número total de especies y con la estructura 
comunitaria de macroinvertebrados realizados en otras zonas estuarinas del Estero Salado y otras épocas de estudio. Así la diversidad faunística bentónica de los ramales estuarinos estudiados, presentaron una baja biodiversidad específica con 11 especies y representados principalmente por los taxones Annelida (Oligochaeta), Arthropoda (Insecta) y Mollusca (Gasteropoda), esta fue poca diversa y menos abundante a la registradas en estudios similares realizados en los ramales estuarinos afectados por actividades humanas como Kennedy, Urdesa Norte y Miraflores durante la época seca (noviembre de 2009) (Cárdena, 2010) donde se registraron 16 especies.

La estación Universidad de Guayaquil presentó la mayor abundancia de organismos debido a la presencia de oligoquetos y el mayor número de especies (7 especies), mientras que estación Aventura Plaza presentó la menor abundancia de organismos, siendo los insectos dípteros los organismos más abundantes y donde se registra el menor número de especies (5 especies). La composición de especies y el predominio de los oligoquetos e insectos fueron similares a los registrados por el Instituto Oceanográfico de la Armada en el estudio de consultoría realizado para el Ministerio del Ambiente en junio de 2012 en los ramales estuarinos entre las ciudadelas Miraflores, Kennedy y Urdesa zonas muy cercanas al área de estudio, donde se registró la presencia de oligoquetos y larvas de insectos (Ministerio del Ambiente, 2012). Sin embargo, la abundancia de organismos registrada en la estación Universidad de Guayaquil fue menor a la registrada en el 2011, donde se registraron 19 especies en la época lluviosa y los invertebrados dominantes fueron los insectos representados por la especie Podura aquatica (Cárdenas et al., 2011).

Los sedimentos del ramal estuarino de la zona Aventura Plaza registró principalmente la presencia de insectos del género Pericoma, insectos de la familia Dolichopodidae y del gasterópodo Heleobia, esta composición no fue similar a la registrada en febrero y marzo del 2012 en la misma zona; donde los oligoquetos y el gasterópodo Melanoides tuberculata fueron los invertebrados predominantes (Cárdenas y Erazo, 2012). Así también la composición, abundancia y diversidad de invertebrados fue distinta a la encontrada en agosto de 1983 por Cruz (1986) quien registró la presencia de 19 especies de bivalvos con predominio de especies estuarinas como Mytella guyanensis, M. strigata, Corbula spp., especies que prefieren sustratos limosos y arenosos en el Estero Salado. Estos cambios de la composición y abundancia de los invertebrados podrían estar asociado a la contaminación generada por la ciudad (Cruz, 2003), que han alterado características físicas, químicas, biológicas del estero como: salinidad, temperatura, concentraciones de oxígeno, sustratos y otros parámetros ambientales, que limitan el asentamiento y colonización de las larvas de invertebrados típicos del manglar como Mytella strigata, Uca sp. , Crassostrea columbiensis, Nephtys singularis, Nereis sp., entre otros. Esto podría atribuirse al aportes de aguas de uso doméstico e industrial que directamente son vertidas sin ningún tipo de tratamiento al estuario y que sólo influyen en la salinidad del estero, siendo este un factor limitante para la supervivencia de especies, permitiendo el desarrollo de especies generalistas tolerantes a la contaminación orgánica e inorgánica, condiciones de hipoxia y aportes de sedimentos alóctonos como los oligoquetos y los insectos dípteros (Pinilla, 2000) que predominan en aguas con contaminación orgánica.

Los patrones de distribución de los macroinvertebrados, la abundancia y diversidad de especies registrados en los sitios de estudio indicarían la presencia de dos comunidades distintas de invertebrados, en la cual la estación Aventura Plaza se caracteriza por la presencia de insectos dípteros mientras que en la estación Universidad de Guayaquil los oligoquetos fueron los organismos dominantes. Dichos organismos están asociados a zonas donde existen efluentes de desechos orgánicos (Goitia et al., 2001), esto refleja que dichos ramales estuarinos continúan siendo perturbados con aportes de Coliformes fecales (Cárdenas et al., 2011), metales pesados, hidrocarburos (Cárdenas et al., 2011; Fernández-Cadena et al., 2014) y otros contaminantes.

La diversidad de especies de los sitios de estudio oscilaron entre 0,1 y 0,6 lo cual corresponde a una diversidad alfa muy baja (0-1) según la tabla de referencia propuesta por Ramírez (2006) fue inferior a la registrada en los ramales estuarinos de Urdesa $(0,469$ bits.org-1) y Universidad de Guayaquil (1,88 bits.org$\left.{ }^{1}\right)$ observados en el 2011 (Cárdenas et al., 2011), esto demostraría que se mantienen los factores estresantes en los ramales estuarinos aledaños a las ciudadelas de Urdesa y Kennedy definidas por la Municipalidad de Guayaquil tales como: decrecimiento de la salinidad, aporte de desechos domésticos, la presencia de detergentes, cloro, grasas, la presencia de metales pesados, hidrocarburos provenientes de aguas no tratadas y vertidas a través del alcantarillado pluvial a la zona estuarina (Hidroestudios, 2003) lo que genera la disminución de la diversidad, el aumento de especies 
oportunistas y el cambio de las comunidades biológicas propias de las zonas estuarinas.

En relación a la variabilidad de la abundancia de invertebrados en los sitios de estudio se observó que la mayor abundancia de invertebrados está asociada a variables como el $\mathrm{pH}$ del agua y la salinidad como se observó en el caso del ramal de la Universidad de Guayaquil donde se registró especies estuarinas como Polymesoda inflata (Russell-Hunter, 2013), Melampus cf carolianus que habita en la arena húmeda entre la vegetación del manglar (Paredes et al., 2005). Sin embargo, también se registró especies de aguas continentales como oligoquetos y gasterópodos como Heleobia sp. y Melanoides tuberculata considerada esta última especie como invasora originaria del África y de Asia (Domínguez y Fernández, 2009) que se ha distribuido también en América del Sur a países como Venezuela, Colombia, Argentina, Uruguay, Brasil (Peso et al., 2010).

Al realizar comparaciones de las comunidades entre las localidades de estudio se observa que la estación Aventura Plaza presenta una comunidad distinta y atípica a una zona estuarina donde predominan los insectos acuáticos y oligoquetos, donde disminuyen la abundancia y la diversidad de las especies. Por lo que se hace necesario la implementación de un programa de monitoreo de la calidad de los efluentes de origen doméstico e industrial en las zonas de estudio y zonas aledañas, dirigido a evaluar parámetros físicos - químicos como: salinidad, temperatura, oxígeno disuelto, $\mathrm{pH}$, detergentes, materia orgánica metales pesados, aceites - grasas, sulfuros e hidrocarburos y parámetros biológicos como coliformes fecales e invertebrados para determinar el impacto de las actividades antropogénicas sobre la salud del ecosistema estuarino. De esta forma se logrará conocer la calidad de las aguas del Estero Salado, conocer las fuentes de los agentes estresores y sus implicaciones en el deterioro del ecosistema acuático. Así también generará información técnica que permitirá a las autoridades ambientales ejecutar medidas legales pertinentes hacia aquellos que incumplen la normativa ambiental ecuatoriana en relación a la calidad ambiental.

\section{6}

Los autores agradecen al Centro de Investigaciones de la Universidad de Especialidades Espíritu Santo por los fondos otorgados para la realización de este estudio, se agradece al apoyo de campo y laboratorio del estudiante de la Escuela de Ciencias Ambientales de la Universidad de Especialidades Espíritu Santo Sr. Julián Pérez por su tiempo en la separación de las muestras biológicas y al Master René Rodríguez Director de la Escuela de Ciencias Ambientales de la Universidad Espíritu Santo por la revisión de este manuscrito y al Dr. Luis Troccoli investigador del Instituto Nacional de Pesca Programa Prometeo por sus recomendaciones en el análisis estadístico.

\section{BIBLIOGRAFÍA}

Ayarza, W., S. Coello, N. Chalen, P. Garcés, M.L. García, F. Ormaza, E. Pérez, F. Pesantes y L. Solórzano. 1993. Estudios Biogeoquímicos de la Sección Urbana del Estero Salado. Ecuador. Revista de Ciencias del Mar y Limnología. Instituto Nacional de Pesca 3 (1):1-19.

Boothroyd, J., H. Ayón, D. Robadue, J. Vásconez, y R. Noboa. 1994. Características de la línea costera del Ecuador y recomendaciones para su manejo. Programa de Manejo de Recursos Costeros. Ecuador.67p.

Bouillon, S. 2011. Storage beneath mangroves. Nature Geoscience 4: 282-283.

CAAM 1996. Desarrollo y Problemática Ambiental del Área del Golfo de Guayaquil. 354 p.

Cárdenas, M. 2010. Efecto de la contaminación hidrocarburífera sobre la estructura comunitaria de macroinvertebrados bentónicos presentes en el sedimento del Estero Salado. Tesis Universidad de Guayaquil.119 p.

Cárdenas, M. y R. Erazo. 2012. Informe técnico de la composición, abundancia, diversidad y distribución de macroinvertebrados bentónicos presentes en los sedimentos de Aventura Plaza durante las pruebas de biorremediación de suelos contaminados con hidrocarburos durante el 2012. 58-74p En: SALADO (ed.). Guayaquil: Escuela Superior Politécnica del Chimborazo. Centro de Servicios Técnicos y de Transferencia Tecnológica Ambiental.164 p.

Cárdenas, M., R. Erazo y J. Mair. 2011. Mediciones de nutrientes, contaminantes, macroinvertebrados y bacterias presentes en el Estero Salado durante enero de 2011. Estudios de contaminantes de los ramales estuarinos que ingresan a la ciudad de Guayaquil Estero Salado. Guayaquil: Heriot Watt University/ Escuela Superior Politécnica del Chimborazo.53 p.

Clark, K. R. y R. M. Warwick. 1994. Change in marine communities: an approach to statistical analysis and interpretation. Natural Environment Research Council. Plymouth, United Kingdom.144 p. 
Clark, K. R. y R. M. Warwick. 2001. Change in marine communities: an approach to statistical analysis and interpretation. Plymouth., United Kingdom. Chapter 6:1-14.

Clarke K. R. y R. N. Gorley. 2006. Primer v6: user manual/ tutorial. PRIMER-E, Plymouth.

Cruz, M. 1983. Bivalvos del Golfo de Guayaquil. Acta Oceanográfica del Pacífico 2 (2): 735-764.

Cruz, M. 1986. Contribución al conocimiento de los Bivalvos en los Esteros del Salado y Cascajal del Golfo de Guayaquil. Interior. Acta Oceanográfica del Pacífico. 3(1):133-148.

Cruz, M., M. González, E. Gualancañay, F. Villamar. 1980. Lista de la fauna sublitoral bentónica del Estero Salado Inferior, Ecuador. Acta Oceanográfica del Pacífico.1 (1):82-96.

Cruz M. 1992. Estado actual del recurso malacológico (Bivalvos y Gasterópodos) de la zona infralitoral del Golfo de Guayaquil. Acta Oceanográfica del Pacífico 7(1): 41-68.

Cruz, M. 2003. Malacofauna bentónica existente en los alrededores de la Ciudad de Guayaquil (EsteroSalado y Río Guayas), Durante 2003. Acta Oceanográfica del Pacífico 12(1): $135-145$.

Cruz, R. y J. Jiménez. 1994. Moluscos asociados a las áreas de manglar de la Costa Pacífica de América Central .Editorial Fundación UNA.180p.

Domínguez, E. y H. R. Fernández. 2009. Macroinvertebrados bentónicos sudamericanos: sistemática y biología, Tucumán- Argentina, Fundación Miguel Lillo. 656p.

EMAG 1978. Recuperación del Estero Salado. Plan de Trabajo. Empresa Municipal de Alcantarillado de Guayaquil. Guayaquil, Ecuador .43 p.

Estrella, T. 2000. Usos del Recurso Agua y Manglares en el Estero de Puerto Hondo, Provincia del Guayas, Ecuador. Tesis de Maestría. Universidad Internacional de Andalucía. Sede Iberoamericana de la Rábida. Huelva- España.119 p.

FAO 1981. Manual of methods in aquatic environment research. Part 8.Ecological assessment of pollution effects. (Guidelines for the FAO (GFCM) / UNEP Joint Coordinated Project on Pollution in the Mediterranean), by J. Stirn Fisheries Technical Paper No 209: 70p.

Fernández -Cadena J. C., S. Andrade, C. L. Silva-Coello y R. De La Iglesia. 2014. Heavy metal concentration in mangrove surface from the north-west coast of South América. Marine Pollution Bulletin. Vol 82 (1-2): 221-226
Goitia, E., R. Ayala, M. Rossberg y A. M. Romero. 2001. Comunidad Bentónica del Río Rocha en relación a la entrada de poluentes (Cochabamba Bolivia). Revista Boliviana de Ecología y Conservación Ambiental.10: 3-6.

Hidroestudios. 2003. Plan Integral de recuperación para el Estero Salado- Fase I .Guayaquil. Informe Técnico preparado para la Muy ilustre Municipalidad de Guayaquil.188p

Holden, R. 1978. Soluciones para el problema de contaminación del Estero Salado-Guayaquil. Facultad de Ingeniería Marítima y Ciencias del Mar. Informe Interno ESPOL. 273-292.

Holthuis, L. B. 1951. A General Revision of the Palaemonidae (Crustacea Decapoda Natantia) of the Americas. II The Subfamily Palaemoninae, Research Associate Allan Hancock Foundation.Ocasional paper 11: 1-332.

Kingston, P. F. 1987. Field effects of platform discharges on benthic macrofauna. Philosophical transactions of the Royal Society of London. B316 (181). 545-565 .

Márquez, B. y M. Jiménez. 2002. Moluscos asociados a las raíces sumergidas del mangle rojo Rhizophora mangle, en el Golfo de Santa Fe, Estado Sucre, Venezuela. Revista de Biología Tropical 50: 1101-1112.

Ministerio Del Ambiente. 2012. Estudio y levantamiento de la batimetría y caracterización del sedimento en el Estero Salado. En: Ecológico, P. G. (ed.). Guayaquil: Ministerio del Ambiente.201 p.

Monserrate, L., J. Medina y P. Calle. 2011. Estudio de condiciones Físicas, Químicas y Biológicas en la Zona Intermareal de Dos Sectores del Estero Salado con Diferente Desarrollo Urbano. Tesis. Escuela Superior Politécnica del Litoral.141p.

Montaño, M. y T. Sanfeliú. 2008. Ecosistema Guayas (Ecuador). Medio ambiente y Sostenibilidad. Introducción. Revista Tecnológica ESPOL. Vol. 21 No. 1: 1-6

Mora, E. y B. Reinoso. 1981. Investigaciones preliminares sobre el estado actual de las poblaciones de ostiones en tres zonas del estuario Interior del Golfo de Guayaquil. Revista Ciencias del Mar y Limnología. Instituto Nacional de Pesca. Vol.1.No1:49-59.

Murray, S. D., A. Siripong y J. Santoro. 1973. Circulation and Salinity distribution in the Río Guayas Estuary, Ecuador. (Estuarine Research) Coastal Studien Institute, L.S.U: 1-23p. 
Olsson, A. A. 1961. Mollusks of the Tropical Eastern Pacific (particularly from the southern half of the Panamic- Pacific Faunal province (Panamá to Perú), Ithaca, Ney York, Paleontological Research Institution ITHACA,N.Y.574 p.

Paredes, C., A. Indacochea, F. Cardoso y K. Ortega. 2005. Familia Ellobiidae (Gastropoda: Archaeopulmonata) en el litoral Revista Peruana de Biología., 12: 69-76.

Pérez, J. 2014. Tipos de mangles presentes en la zona de Aventura Plaza y la Universidad de Guayaquil. Informe Técnico de Salidas de Campo.

Pérez, J. 2012. Caracterizacion del componente de Flora del Estero Salado en los ramales con diferentes tipos de desarrollo urbano. Informe técnico preparado para Ecobiotec del Ecuador y Ministerio del Ambiente.276p.

Pesantes, F. 1975. Distribución de las propiedades físicas y químicas del Golfo de Guayaquil. Revista de la Comisión Permanente del Pacífico Sur. Guayaquil.3:21-37

Peso, J., R. Vogler y N. Pividori. 2010. Primer Registro del Gasterópodo invasor (Gastropoda,Thiaridae) en el río Uruguay (Argentina -Brasil). Comunicaciones de la sociedad Malacológica del Uruguay.231-236 pp.

Pinilla, G. 2000. Indicadores biológicos en ecosistemas acuáticos continentales de Colombia. Bogotá, Colombia. Editorial. Fundación Universidad de Bogotá Jorge Tadeo Lozano.51p.

Pires 2000. Estudios de Prefactibilidad, factibilidad y Selección de la mejor alternativa del Plan Integral de la recuperación del Estero Salado. Informe Final Parte II. Guayaquil. Capítulo 8: 1-49.

Ramírez, A. 2006. Métodos de muestreo y análisis de poblaciones y comunidades, Bogotá, Facultad de Ciencias Ambientales y Rurales de la Pontificia Universidad Javeriana.271p.

Rodríguez, A. 2005. Contaminación por Residuo de Hidrocarburo del Petróleo en el Puerto Marítimo de Guayaquil y su área de influencia debido a actividades navieras, período 1984 al 2004. Acta Oceanográfica del Pacífico. Guayaquil. Vol. 103 No. 1.

Roldan, G. 1988. Guía para el estudio de macroinvertebrados acuáticos del Departamento de Antioquia, BogotáColombia. Fondo Fen Colombia/Colciencias/Universidad de Antioquia.

Russell-Hunter, W. D. 2013. The Mollusca. Ecology. Volumen 6. New York-USA.

Shannon, C. E. y W. Weaver, 1963. The mathematical theory of communication. University of Illinois Press. Urbana, Illinois .125p

Siguencia, R. 2010. Niveles de Coliformes Totales Y Escherichia Coli en bivalvos de interés comercial Ostrea columbiensis y Mytella guyanensis (Molusca: Bivalvia) como bioindicador de contaminación microbiológica en el Estero Puerto Hondo, Provincia del Guayas - Ecuador. Tesis de Maestría, Universidad de Guayaquil, Ecuador. 48 p.

Stevenson, M. 1981. Variaciones Estacionales en el Golfo de Guayaquil, un estuario tropical. Boletín Científico y técnico Guayaquil: Instituto Nacional de Pesca.133p.

Thiakos, V. 2000. Investigation of the Hydrocarbon Pollution by Diffuse and point sources in the Gulf of Guayaquil, in the Republic of Ecuador. Thesis. Heriot Watt University.84p.

Twilley, R. R. 1989. Impacts of Shrimp mariculture Practices on the Ecology of Coastal Ecosystems in Ecuado, p: 91120. En: S. Olsen and L. Arriaga (Eds.). Establishing a Sustainable Shrimp mariculture Industry in Ecuador. University of Rhode Island,Technical Report Series TR-E-6.

Valverde, F. D. y J. Pérez. 2012. La biodiversidad vegetal como capital natural de la sostenibilidad en la costa ecuatoriana, Guayaquil, M: I: Municipalidad de Santiago de Guayaquil.797p

Zambrano, N. 2007. Plan de Manejo del Bosque Protector Estero Salado Norte. Programa de Manejo de Recursos Costeros. 79p.

Para citar este artículo: Cárdenas-Calle, M. y Mair, J. 2014. Caracterización de macroinvertebrados bentónicos de dos ramales estuarinos afectados por la actividad industrial, Estero Salado-Ecuador. Revista Intropica 9: 118 - 128 